\title{
VR as a choice: what drives learners' technology acceptance?
}

\author{
Sean M. Noble ${ }^{*}$ (D, Jason D. Saville* and Lori L. Foster ${ }^{*}$
}

\section{*Correspondence:}

smnoble2@ncsu.edu;

jdsavill@ncsu.edu;

Ilfoster@ncsu.edu

Department of Psychology,

North Carolina State

University, Poe Hall, 2310

Stinson Dr, Raleigh, NC

27695, USA

\begin{abstract}
Post-secondary institutions are investing in and utilizing virtual reality (VR) for many educational purposes, including as a discretionary learning tool. Institutions such as vocational schools, community colleges, and universities need to understand what psychological factors drive students' acceptance of VR for learning in discretionary contexts. The Unified Theory of Acceptance and Use of Technology (UTAUT; Venkatesh et al. in MIS Quarterly 27:425-478, 2003) offers a theoretical framework for understanding students' receptivity to VR for learning. Undergraduate university students $(N=300)$ read a description of VR and video training mediums, then indicated which they would choose to learn a novel task. Three psychological variables-performance expectancy, effort expectancy, and social influence- - tended to be related to acceptance of $V R$, which was measured in two ways: (a) rated intentions to use VR and (b) preference for VR over a video-based alternative. Relative weight analyses compared the importance of the three predictors and revealed that performance expectancy tended to be the most influential antecedent of VR acceptance.
\end{abstract}

Keywords: Technology acceptance, Virtual reality, Post-secondary education, Relative weight analysis, UTAUT

\section{Introduction}

Colleges and universities across the globe strive to ensure the students enrolled have access and exposure to technologies that will help them learn and later transition to the world of work. While technology may be introduced in the classroom, often it is not. Rather, usage is often discretionary. Consider, for example, a new piece of hardware or software in an on-campus makerspace studio. Students-including those with no experience with the technology-make the decision to use or to not use this resource. This raises interesting questions about what drives acceptance of a discretionary learning technology.

In this context, virtual reality (VR) is especially interesting because of its potential and availability. Post-secondary institutions have developed an interest in VR due to its ability to enhance certain learning outcomes (e.g., Jensen \& Konradsen, 2018; Makransky et al., 2019; Man, 2018; Siu et al., 2016). While institutions have begun introducing VR in the classroom (FSR Education, 2018; Schaffhauser, 2019), VR has also been introduced outside the classroom as a discretionary learning tool (e.g., Forest et al., 2014). Evidence author(s) and the source, provide a link to the Creative Commons licence, and indicate if changes were made. The images or other third party material in this article are included in the article's Creative Commons licence, unless indicated otherwise in a credit line to the material. If material is not included in the article's Creative Commons licence and your intended use is not permitted by statutory regulation or exceeds the permitted use, you will need to obtain permission directly from the copyright holder. To view a copy of this licence, visit http:// creativecommons.org/licenses/by/4.0/. 
suggests that students may be underutilizing this resource (Margaryan et al., 2011; Pak et al., 2017). The introduction of VR to post-secondary institutions is still relatively new, and research is needed to unpack students' views of VR for learning, including the characteristics that drive differences in VR acceptance. The purpose of this study is to examine: (a) cognitive beliefs that prompt students to choose or to avoid the discretionary use of VR for learning and (b) the relative importance of these beliefs.

\section{Virtual reality}

Howard (2018) defines VR as the "computer-simulation of an environment that imitates a physical presence in real or imagined worlds" (p. 4). While VR has existed for decades, the usage of VR in education has become increasingly common because of the recent development of affordable, commercially available head-mounted display (HMD) devices which enable greater immersion than ever before. HMDs, such as the Vive (HTC Corporation, 2021) or Oculus Rift (Oculus VR, LLC, 2021), are visual display monitors worn directly over the user's eyes. While experiencing HMD VR, the entirety of the user's vision is encompassed within the virtual environment. HMDs traditionally display 360-degree video output, allowing users to move their head freely and utilize their sight akin to how they would look around to see in a real environment.

HMD VR has been applied to learning content domains such as procedural-practical knowledge, declarative knowledge, analytical problem-solving, interpersonal skills, behavioral impacts, and language learning (Radianti et al., 2020). A systematic review of VR research found that HMD VR is effective for visual and spatial learning (Jensen \& Konradsen, 2018). Howard (2018) conducted a meta-analysis of more than 191 studies in order to compare the effects of HMD VR to computer-monitor VR. Results indicated that HMDs led to greater cognitive and emotional outcomes compared to computer monitor VR. Rupp et al. (2019) also found that, for students who had previously never utilized VR, using HMD VR resulted in greater interest in the subject matter than when using less immersive VR.

For these reasons, VR devices have already found a plethora of uses for learning. VR has been used for practicing clinical nursing techniques, visualizing astronomy concepts and stage design, recreating historical events, simulating space walks, and engaging in leadership exercises (FSR Education, 2018; Schaffhauser, 2019). Some post-secondary institutions are already developing majors and courses to educate students on VR through the use of VR labs (Jones, 2018; Le, 2018; University of Washington, 2021). These investments and developments are meant to both educate students using VR and to educate them about VR itself. To that end, post-secondary institutions are introducing VR to students not only as a prescribed learning tool, but also by making it available for use outside of the context of a course, such as in labs, libraries, and other learning spaces (e.g., Forest et al., 2014). In these venues, students can access VR at their discretion to learn about things related to their intended career, a major they are considering, their hobbies, or simply their curiosities. For example, students considering a major in architecture could independently go to their library's VR facility to try their hand at AutoCAD, those interested in the medical field could attempt suturing, and those about to embark on a spring break trip abroad could seek out campus VR resources to practice cross-cultural interaction. 


\section{Student acceptance and usage of $V R$}

Despite the educational benefits of providing students discretionary access to VR, research suggests students are underutilizing VR as a discretionary learning tool. Students may be aware that VR resources exist on their campuses, such as learning opportunities, training programs, and open-access makerspace labs, and yet still decide not to use these resources. Margaryan et al. (2011) administered a series of quantitative surveys to investigate the extent to which university students utilized various technologies for learning outside of a course. Their findings indicated that students tend to gravitate toward technologies they are already familiar with. Concerning the use of VR, the researchers found that $76 \%$ of the 160 students sampled reported "never" using simulations or games in informal learning scenarios. While Margaryan et al. (2011) documented such usage patterns prior to the VR "boom" of 2012, more recent research suggests this lack of exposure to VR among students persists. Pak et al. (2017) asked 239 university students to use a 5-point scale to rate the extent to which they had experience with various VR devices, with higher ratings representing greater usage. Responses revealed a relatively low utilization rate, with mean scores ranging from 1.00 to 1.85 for all VR devices listed on the survey.

\section{The unified theory of acceptance and use of technology}

Current usage patterns lead to important questions about what encourages and discourages VR acceptance. User acceptance models provide a helpful way to understand people's receptivity toward a variety of technologies, including VR. These models assess individuals' acceptance of technologies by measuring the cognitive factors associated with behavioral intention to use a given technology. Importantly, user acceptance models can shed light on what drives intentions to use a technology regardless of prior exposure. Just as people range in their acceptance of technologies with which they have first-hand experience, they also vary with respect to their intentions to use technologies they have only heard about. Even those with no prior exposure can imagine what there is to gain from using a new tool, and likely hold assumptions about the effort required for adoption or the social pressure to use it. Accordingly, user acceptance models offer ways to understand the intentions pertaining to both more and less familiar technologies.

More than 15 years ago, Venkatesh et al. (2003) integrated the most prominent user acceptance models of their era, including the Technology Acceptance Model (TAM; Davis, 1989; Davis et al., 1989), the Technology Acceptance Model 2 (TAM2; Venkatesh \& Davis, 2000), and the Theory of Planned Behavior (TPB; Ajzen, 1991), to form the Unified Theory of Acceptance and Use of Technology (UTAUT). In many ways, the UTAUT represents the direct continuation of the TAM and TAM2. The TAM, TAM2, and UTAUT all suggest that cognitive beliefs regarding a technology's ability to improve one's performance are the primary predictors of behavioral intention to use that technology (Davis et al., 1989; Venkatesh \& Davis, 2000; Venkatesh et al., 2003). All three models suggest that perceived usefulness (called "performance expectancy" in the UTAUT) and perceived ease of use (called "effort expectancy" in the UTAUT) drive technology acceptance. TAM2 incorporated the construct of subjective norms (called "social influence" in the UTAUT) as an additional predictor, suggesting that important others' beliefs contribute to behavioral intention to use a technology. UTAUT included these 
three predictors, as well as similar constructs from other behavioral intention models, to develop items for an integrated model of technology acceptance (Venkatesh et al., 2003).

To verify the UTAUT's utility above and beyond its predecessors, Venkatesh et al. (2003) conducted a longitudinal study which determined that the UTAUT accounted for a robust amount of variance $\left(R^{2}=0.69\right)$ in behavioral usage of technology, more than any of the models that had been synthesized to create the UTAUT. Support for the validity of the UTAUT has been demonstrated by many studies since its creation (see Venkatesh et al., 2016). Accordingly, the present study uses UTAUT to understand students' acceptance of VR for learning.

The first UTAUT predictor is performance expectancy (see Venkatesh et al., 2003), defined as the degree to which individuals perceive that using a technology will increase their performance. In the context of VR for learning, performance expectancy refers to students' anticipation that VR will help them achieve the goal they are aiming for, such as improved mastery of a given learning topic. In models that preceded the UTAUT, constructs representing types of performance expectancy (e.g., perceived usefulness, extrinsic motivation, job-fit, relative advantage, and outcome expectations) strongly predicted behavioral intention to use a technology (Venkatesh et al., 2003). Simply put, people are more likely to accept a new technology if they feel that the effort required to use it will "pay off" in terms of useful gains, and are motivated to avoid using a technology which would fail to provide utility.

Whereas performance expectancy refers to the "benefit" side of the cost-benefit equation, effort expectancy relates to the psychological cost of using a new system. Effort expectancy is the ease of use associated with a technology (Venkatesh et al., 2003). Venkatesh et al. (2003) note that similar constructs (e.g., perceived ease of use, complexity) were previously found to predict behavioral intention, particularly when individuals first started using a new technology. Hobfoll's (1989) conservation of resources theory indicates that individuals are motivated to conserve resources (i.e., objects, characteristics, conditions, or energies) in the face of stressors which threaten these resources. Technologies that require less effort or energy are thus more likely to be accepted.

Social influence is the degree to which people perceive that individuals important to them support using the technology. Social influence is similar to some variables in models preceding UTAUT, such as subjective norms, social factors, and image. Social influence in the UTAUT originates from the notion that human beings are social creatures, and that individuals' behaviors are influenced by perceptions of how others will view them for having used (or not used) the technology in question (Venkatesh et al., 2003). Bandura's (1977) Social Learning Theory postulates that individuals will be more likely to engage in behavior when it is encouraged and supported by referent others, such as one's colleagues, professors, peers, supervisors, or post-secondary institution administrators. Similarly, Ajzen's (1991) Theory of Planned Behavior, a theoretical contributor to the UTAUT, posits that perceived social pressure to perform a behavior influences intentions to perform said behavior.

Extant research has analyzed these predictors and other similar constructs, providing early insights into acceptance of VR for learning. Makransky and Lilleholt (2018) allowed university students in a lab setting to experience a learning simulation through HMD VR, as well as computer monitor VR, before completing a series 
of surveys. Researchers used structural equation modeling (SEM) to examine, among other criteria, the antecedents of perceived learning and behavioral intention to use VR for learning. Results supported an aggregate variable of usefulness (i.e., performance expectancy) and perceived ease of use (i.e., effort expectancy) as a predictor of behavioral intention to use a given technology, mediated by a variety of factors. A year later, Shen et al. (2019) published a complementary study examining behavioral intention to use VR for learning. Students were shown an online video, viewable using their computer screen, which described how VR could be used for learning in a classroom context. Participants then completed a survey battery assessing, among other constructs, performance expectancy, effort expectancy, social influence, and behavioral intention to use VR for learning. Utilizing SEM, Shen et al. (2019) found that performance expectancy, effort expectancy, and social influence were all significant predictors of behavioral intention to use VR for learning.

While findings of both studies add to our understanding, questions about acceptance of VR for learning remain. Notably, neither of these studies examined VR for learning in a discretionary context, such as when VR is available for optional use outside of the classroom. In addition, both studies relied on a single outcome variable to measure acceptance-namely, rated intentions to use VR in the future. While behavioral intention to use VR is a useful way to conceptualize acceptance of VR, there is value in measuring acceptance in other ways as well. Measuring acceptance in multiple ways ensures findings are not limited or artifactually tied to a single way of conceptualizing and assessing VR acceptance.

In short, the present study contributes to the literature on acceptance of VR for learning and expands on prior research in three ways, by (a) examining the UTAUT model when the use of VR is discretionary, (b) testing this model across two different indicators of VR acceptance, and (c) examining the relative importance of the antecedents of VR acceptance, which we discuss in greater detail below.

Hypotheses In order to best examine students' receptivity to VR for learning, this study uniquely looks at acceptance in two ways: (a) behavioral intention to use VR for learning and (b) preference to use VR for learning over an alternative method of learning (namely, video-based training). In accordance with the UTAUT, students are expected to report greater acceptance of VR for learning when they believe VR will enable them to successfully perform the task under consideration (i.e., high performance expectancy) and when they believe they will be able to successfully use the technology (i.e., high effort expectancy). Students who sense an urging from important referent others (i.e., high social influence) are also expected to report greater acceptance of VR for learning.

Hypothesis 1 Performance expectancy will positively predict acceptance of VR for learning.

H1a Performance expectancy will positively predict behavioral intention to use VR.

$H 1 b$ Performance expectancy will positively predict preference for VR over a videobased training medium. 
Hypothesis 2 Effort expectancy will positively predict acceptance of VR for learning.

H2a Effort expectancy will positively predict behavioral intention to use VR.

$H 2 b$ Effort expectancy will positively predict preference for VR over a video-based training medium.

Hypothesis 3 Social influence will positively predict acceptance of VR for learning.

H3a Social influence will positively predict behavioral intention to use VR.

$H 3 b$ Social influence will positively predict preference for VR over a video-based training medium.

In addition to testing whether each of these three hypothesized variables uniquely predict VR acceptance, there is value in rigorously examining their relative importance. Compelling empirical examinations of this matter are scant. This is surprising because the relative importance of various drivers of technology acceptance has been a topic of discussion for years. Foundational research on the development of the TAM (Davis et al., 1989), TAM2 (Venkatesh \& Davis, 2000), and UTAUT (Venkatesh et al., 2003) suggested that performance expectancy was the strongest of these three antecedents and used regression weights as evidence to support these suggestions. Dwivedi et al. (2011) later conducted a meta-analysis on 162 articles on the UTAUT using meta-analytic structural equation modeling (MASEM). Based on their findings, performance expectancy $(\beta=0.32)$ was reported as the strongest predictor of behavioral intention to use a system, followed by effort expectancy $(\beta=0.27)$, and finally social influence $(\beta=0.17)$.

However, while regression weights, SEM, and path coefficients have historically been used to draw conclusions, these methods do not sufficiently test the relative importance of performance expectancy, effort expectancy, and social influence. The reason such methods are insufficient is because they fail to account simultaneously for both the effect of the predictor variable itself and the effect of the predictor in combination with the other predictor variables in the model (Johnson, 2000). The present study addresses this gap with an approach rarely, if ever, employed in the technology acceptance literature to date: Johnson's (2000) relative weight analysis (RWA). Unlike hierarchical linear regression or SEM, RWA accounts for the effect of each predictor on the criterion individually as well as their effects in tandem. In this way, RWA accurately partitions the percentage of variance accounted for by each predictor on the criterion (Tonidandel et al., 2009), producing a rigorous index of each predictor's relative influence or importance.

Davis et al. (1989) postulated that performance expectancy is more cognitively proximal than effort expectancy regarding one's performance goals and therefore the stronger predictor of behavioral intentions to use a technology. However, Davis et al's (1989) explanation was post-hoc and based on inductive rather than deductive reasoning. Furthermore, subsequent expansion of the TAM in the form of the TAM2 (Davis \& Venkatesh, 2000) and UTAUT (Venkatesh et al., 2003) focused on expanding the breadth of variables within the model rather than the theoretical reasoning behind the relative weight of its predictors. Because there is both little theoretical and empirical basis for 
hypotheses concerning the relative weight of performance expectancy, effort expectancy, and social influence, we approach the question of their relative importance in an exploratory fashion. We examine as a research question whether performance expectancy, effort expectancy, and social influence differentially influence students' acceptance of VR.

Research Question 1: What is the relative influence of performance expectancy, effort expectancy and social influence on acceptance of VR for learning?

RQ1a: What is the relative influence of performance expectancy, effort expectancy and social influence on behavioral intention to use VR?

RQ1b: What is the relative influence of performance expectancy, effort expectancy and social influence on preference for VR over a video-based training medium?

\section{Method}

Participants

We recruited participants $(N=325)$ enrolled in introductory psychology courses at a university in the southeastern United States which offers free access to VR resources at various places on campus, including but not limited to the library. Participants represented the population of interest in that they were post-secondary students, with discretionary access to campus VR resources, who ranged with respect to their prior experience using VR. Participants were enrolled in psychology courses as a requirement for a wide variety of different majors. As a course requirement, they were given the option to spend time participating in research studies, or to complete an alternative assignment at the instructor's discretion. Students wishing to participate in research selected the current study after viewing it listed amongst other studies on a web-based system designed to connect students with research participation opportunities. Participants were required to be 18 years or older to take part in the study. Those choosing to participate were compensated with course research credit.

Concerning gender, $57.0 \%$ of the final sample identified as female, $42.7 \%$ as male, and $0.3 \%$ as transgender. In terms of race/ethnicity, $76.3 \%$ of participants self-identified as Caucasian or White, $12.0 \%$ as Asian, $5.0 \%$ as Black or African American, 3.0\% as Hispanic or Latino, $1.0 \%$ self-identified as Native American or Pacific Islander, and 2.6\% preferred not to say. Ages ranged from 18 to $32(M=19.00, S D=1.49)$.

\section{Design and procedure}

This study includes three predictors: performance expectancy, effort expectancy, and social influence. The two criteria are behavioral intention to use VR and preference for VR over video to learn a novel task.

Participants began by reading an informed consent form explaining that they were taking part in a study examining learning technology preferences. Those consenting then read the brief article shown in Appendix A describing two mediums, VR and video (i.e., YouTube), as different training technologies used to teach and learn new things. We described both mediums in a manner reflective of how they are often described in public discourse (e.g., Weirs, 2020; Zielinski, 2021); VR was presented as a new technology with interesting possibilities, whereas YouTube was described as a common and useful medium of training. Participants were then asked to imagine they were learning a novel 
task as described in Appendix B. Specifically, they were prompted to imagine the need to learn how to suture-a common task for medical professionals to stitch together a wound or incision. We chose suturing as the example task because it would be novel for participants rather than a reinforcement of prior learning and because it is commonly taught in VR (Amirian et al., 2014; Kazemi et al., 2010; Samadbeik et al., 2018). Given that suturing is typically performed by trained medical professionals, we reasoned that such a learning objective would be new to most students taking a psychology course.

Through the scenario shown in Appendix B, we informed participants that the task requires technical knowledge which can be learned on campus through either VR or video resources. Participants were informed that in this scenario they would be taught how to use the VR system or how to properly search YouTube for videos technologies in question until they were comfortable with their chosen medium, and would have however long they liked to learn with either medium. After reading through this prompt, they reported their preference for VR or video to learn the new task. After reporting their learning medium preference, all participants were given a prompt which asked them to imagine that their university started a training program which gives students access to VR technologies to learn new skills. Participants were then asked detailed questions about their perceptions of VR as a training medium. As shown in Appendix $C$, survey items assessed performance expectancy, effort expectancy, social influence, and behavioral intention to use VR, given the opportunity. It is important to emphasize that these survey questions only pertained to students' thoughts on VR for learning, not video, regardless of which preference they had previously indicated. Finally, participants reported their demographic information, including their previous hours of experience with VR, and were debriefed, thanked, and dismissed.

\section{Measures}

To assess the variables examined in this study, we utilized both a dichotomous measure of preference for VR or video in a vignette scenario as well as a survey battery of Likerttype items to measure performance expectancy, effort expectancy, social influence, and behavioral intention to use VR. The vignette and dichotomous choice measure can be found in Appendix B. The Likert-type items were developed directly from measures provided by Venkatesh et al. (2003). The items have been previously analyzed by Venkatesh et al. (2003) using bootstrapping to test partial least square models. Venkatesh et al.s (2003) items are frequently used to assess UTAUT constructs with the items adapted to reference technologies researchers seek to target (e.g., Gupta et al., 2008). Their factor structure has been previously validated via confirmatory factor analysis when adapted to the use of VR for learning (Shen et al., 2019).

In the current study, Venkatesh et al. (2003) items were modified as follows. The word "system" from the original items was replaced with "VR" or "VR for learning" in this study. The full list of scale items can be found in Appendix C. As indicated in Appendix $\mathrm{C}$, items were prefaced with a prompt which asked participants to "Imagine [university] starts a training program for students. The program gives students access to VR technologies to train new skills. Please respond to each of the following items with that scenario in mind." All Likert-type response scales were 7-point, ranging from Strongly disagree 
(1) to Strongly agree (7). All scales exhibited accepted levels of internal consistency, as indicated by the Cronbach's alpha values reported below. Each scale is as follows.

\section{Performance expectancy}

Four items ( $\alpha=0.89$ ) assessed performance expectancy. An example item is, "If I use VR, I will increase my chances of learning" (Venkatesh et al., 2003).

\section{Effort expectancy}

Four items $(\alpha=0.87)$ assessed effort expectancy. An example item is, "I would find VR easy to use" (Venkatesh et al., 2003).

\section{Social influence}

Four items $(\alpha=0.78)$ assessed participants' perceptions of social influence supporting their use of VR. An example item is, "People who influence my behavior think that I should use VR for training” (Venkatesh et al., 2003).

\section{Behavioral intention}

Three items $(\alpha=0.92)$ were included to assess participants' behavioral intention to use VR. An example item is, "I predict I would use the VR system for learning in the first 3 months" (Venkatesh et al., 2003).

\section{VR or video preference}

Preference to use VR or video to learn a novel task was measured by a dichotomous self-report. To make a realistic comparison of learning options available to students, we operationalized video learning as YouTube. YouTube is an online video platform which allows students to freely select an instructional video from those uploaded by YouTube community members in order to learn the referenced material. After reading the description of VR and video for training shown in Appendix A, participants read the vignette shown in Appendix B, which asked them to imagine that they needed to learn how to suture and to choose either video of VR to learn the technique. Choice of video was coded as 0 . Choice of VR was coded as 1 .

\section{VR experience}

One item was included to assess participants' prior experience with VR. This item read, "How many hours of experience have you had with VR?".

\section{Data analysis}

Prior to addressing hypotheses, data were cleaned and coded. The survey recorded 325 responses. To account for careless responding (see Meade \& Craig, 2012) we included an instructed response item asking individuals to mark "Strongly disagree." Additionally, at the end of the survey we asked respondents if they answered the survey honestly. Participants who failed to mark "Strongly disagree" or indicated that they failed to answer the survey honestly were removed from the analyses. All other participant data $(N=300)$ were included in the focal analyses. All scales were found to have an acceptable internal consistency, as indicated by Cronbach's alpha of greater than 0.7 (Cortina, 1993; see 
Table 1). All Likert-type measures were checked for skewness and kurtosis and found to be within acceptable parameters. To test the hypotheses and research questions, we utilized a combination of bivariate correlation, logistic regression, multiple regression, multiple moderated regression, and RWA.

\section{Results}

Table 1 shows the means, standard deviations, and correlations among this study's variables. As shown in Table 1, the sample reported an average of $4.47 \mathrm{~h}$ of prior experience with VR with a large standard deviation $(S D=15.15)$ clearly influenced by one participant who reported $100 \mathrm{~h}$ and one participant who reported $200 \mathrm{~h}$ of experience; both of these values were substantially higher than the experience levels indicated by the other participants. Just over half (51\%) of the respondents indicated no prior experience, resulting in a median and modal response of $0 \mathrm{~h}$. VR experience was not significantly related to class standing, $F(4,288)=1.87, p=0.12$. As shown in Table 1 , VR experience was positively related to effort expectancy, and social influence and was not significantly related to either of this study's criterion variables of behavioral intention to use VR or preference for VR over video. Table 1 also shows the correlations among this study's predictor and criterion variables. The moderate $(r=0.36)$ correlation between behavioral intention to use VR and preference for VR over video suggests that the two criterion variables indeed tap into related but distinct aspects of VR acceptance, as intended.

Hypotheses 1, 2, and 3 predicted that performance expectancy, effort expectancy, and social influence would predict VR acceptance. First, behavioral intention to use VR was examined. As shown in Table 1, each of the three hypothesized predictors were significantly correlated with intentions to use VR. Next, this criterion was regressed onto performance expectancy, effort expectancy, and social influence. As shown in Table 2, the three predictors together accounted for $28 \%$ of the variance in intentions to use VR $(p<0.001)$, and each predictor uniquely and significantly contributed to the overall model. Thus, hypotheses H1a, H2a, and H3a were supported.

Additionally, we conducted a second post-hoc exploratory analysis to determine if participants' previous hours of VR experience moderated the relationship between the predictor variables and behavioral intention. A moderated multiple linear regression analysis suggested that VR experience was not a significant moderator of the relationships between any of the predictor variables and behavioral intention to use VR. This

Table 1 Correlations, means, and standard deviations

\begin{tabular}{|c|c|c|c|c|c|c|c|}
\hline Measure & $\mathbf{M}(S D)$ & 1 & 2 & 3 & 4 & 5 & 6 \\
\hline 1. Performance expectancy & $5.18(1.04)$ & $(0.89)$ & & & & & \\
\hline 2. Effort expectancy & $5.04(1.08)$ & $0.52^{* *}$ & $(0.87)$ & & & & \\
\hline 3. Social influence & $4.02(1.04)$ & $0.34^{* *}$ & $0.27^{* *}$ & $(0.78)$ & & & \\
\hline 4. Behavioral intention to use VR & $4.56(1.20)$ & $0.49^{* *}$ & $0.38^{* *}$ & $0.32^{* *}$ & $(0.92)$ & & \\
\hline 5. Preference for VR & $0.65(0.48)$ & $0.49 * *$ & $0.26^{* *}$ & $0.24^{* *}$ & $0.36^{* *}$ & - & \\
\hline 6. VR experience & $4.47^{\mathrm{a}}(15.15)$ & 0.05 & $0.17^{* *}$ & $0.13^{*}$ & 0.10 & -0.02 & - \\
\hline
\end{tabular}

$(N=300),{ }^{*} p<0.05,{ }^{* *} p<0.01$. Cronbach's alphas are shown on the diagonal. Preference for VR was coded as 0 for video preference and 1 for preference for VR

${ }^{a}$ The mean VR experience value was influenced by two outliers; the median and mode for VR experience were both 0 
Table 2 Summary of the multiple regression model predicting behavioral intention to Use VR

\begin{tabular}{llllllll}
\hline & $R^{2}$ & $p$ & $N$ & $F$ & $d f$ & & \\
\hline Overall model & 0.28 & $<0.001$ & 300 & 41.12 & 3 & & \\
\hline & $\beta$ & $p$ & $B$ & $t$ & $S E$ & $\begin{array}{l}\text { Relative } \\
\text { weight (\%) }\end{array}$ & Relative weight Cl \\
\hline Performance expectancy & 0.35 & $<0.001$ & 0.41 & 5.97 & 0.35 & 54 & {$[0.08,0.22]$} \\
Effort expectancy & 0.16 & $<0.01$ & 0.17 & 2.68 & 0.16 & 26 & {$[0.02,0.13]$} \\
Social influence & 0.16 & $<0.01$ & 0.19 & 3.04 & 0.16 & 20 & {$[0.01,0.11]$} \\
\hline
\end{tabular}

Table 3 Summary of the logistic regression model predicting preference to use VR

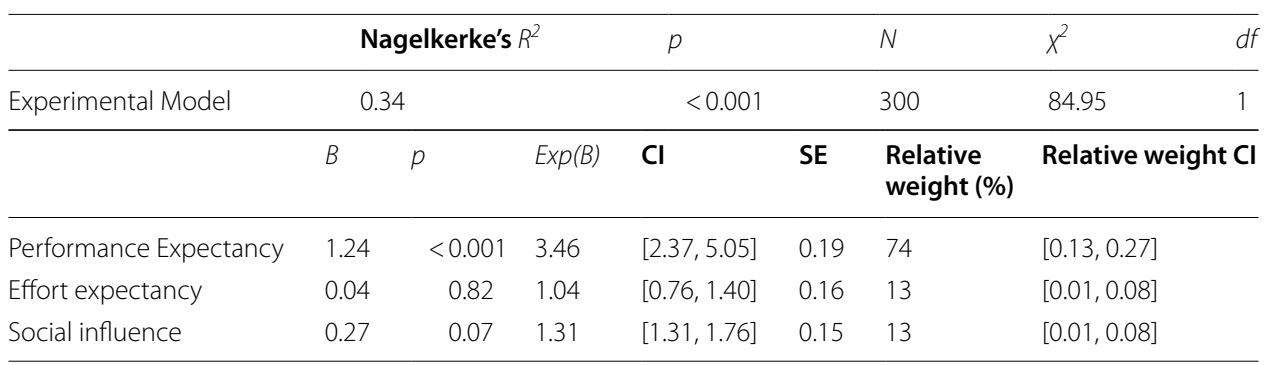

non-significant finding occurred regardless of whether the VR experience outliers were included in the analysis.

To determine the proportion of the explained variance in behavioral intention contributed by the predictors, as asked by RQ1a, we conducted a multiple linear regression RWA using the multiple regression program of the RWA-WEB tool (Tonidandel \& LeBreton, 2015). The analyses utilized 10,000 bootstrapped replications at a $95 \%$ confidence interval (CI). As shown in Table 2, results indicated that performance expectancy accounted for $54 \%$ of the model variance, effort expectancy accounted for $26 \%$, and social influence accounted for $20 \%$. Confidence interval tests of significance using RWAWEB tested whether these relative weights were significantly different from each other. Results indicated that only performance expectancy and social influence were significantly different from one another, $95 \% \mathrm{CI}=[-0.19,-0.01]$. Thus, RQ1a was assessed, and the data indicated that while performance expectancy was more important than effort expectancy and social influence, the latter two variables did not significantly differ between each other in their importance.

Next, preference to use VR for learning was examined as a second index of technology acceptance. Overall, $65 \%$ of participants $(n=196)$ chose VR for learning, and the remaining $35 \%(n=104)$ chose video when given the option. A chi-square goodness of fit test confirmed that students were significantly more likely to choose VR than video, $\chi^{2}(1)=28.2, p<0.001$. The point biserial correlations shown in Table 1 indicate that each of the three hypothesized predictors were significantly correlated with preference for VR, providing initial support for H1b, H2b, and H3b. A logistic regression was conducted to examine the three predictors in tandem. As shown in Table 3, results indicated that the overall model was an improved fit over the null model, with the three predictors together explaining more than one-third of the variance in preference for VR, Nagelkerke $R^{2}=0.34, p<0.001$. Whereas performance expectancy 
significantly predicted preference for VR in the model, neither effort expectancy nor social influence reached statistical significance. Thus, H1b was fully supported, while $\mathrm{H} 2 \mathrm{~b}$ and H3b were partially supported through point biserial correlations in Table 1, but not in the logistic regression analysis in Table 3.

We then conducted another series of post-hoc exploratory analyses to examine if prior VR experience (with and without outliers) moderated the relationship between the predictor variables and preference for VR. These moderated logistic regression analyses suggested that VR experience was not a significant moderator of the relationships between any of the predictor variables and preference for VR.

To examine RQ1b and thereby determine the relative influence of the three predictors on students' preference for VR, we conducted a logistic RWA using RWA-WEB (Tonidandel \& LeBreton, 2015). The analysis utilized 10,000 bootstrapped replications at a 95\% confidence interval. As shown in Table 3, results indicated that performance expectancy accounted for $74 \%$ of the explained variance, effort expectancy accounted for $13 \%$, and social influence accounted for the remaining $13 \%$. We ran confidence interval tests of significance using RWA-WEB to again determine whether these relative weights were significantly different. Results again highlighted the relative importance of performance expectancy, indicating that performance expectancy was significantly more influential than both effort expectancy, 95\% CI [- 0.24, -0.09 ] and social influence, 95\% CI $[-0.24,-0.08]$, which did not significantly differ from each other, as suggested by their identical relative weights.

\section{Discussion}

VR is being deployed in post-secondary institutions worldwide, either embedded within specific educational programs or accessed at the discretion of students. Institutions need to understand the factors that influence the degree to which students will seek out and use VR resources. Those implementing VR in their institutions should keep in mind that a significant percentage of students may not have prior experience with the technology, and that the formation of beliefs surrounding the use of VR is not predicated on having used VR previously. The data in this study were collected from students attending a university that offers discretionary access to VR free of charge. As examples, the university library offers free access for students to borrow virtual reality equipment, access workshops and online modules for topics such as 3D environment design, reserve space and time to play virtual reality games or develop content, and more. Even with opportunities such as these at their fingertips (which does not necessarily suggest awareness), approximately half of the respondents reported no experience with VR. Technology acceptance models are often deployed in research under the assumption that respondents have had a brief interaction with the technology (e.g., Davis et al., 1989). The current study exemplifies that the need to understand the factors that influence students' intentions to seek out and use VR resources extends to both students with and without prior VR experience. Additionally, it suggests an opportunity to increase students' engagement with VR might lie in changing cognitive beliefs about VR for learning: namely, how it can help them learn, how they can learn to use it, and how the institution supports its use. 


\section{Theoretical and methodological implications}

While prior studies have examined performance expectancy, effort expectancy, and social influence as predictors of behavioral intention to use VR (Makransky \& Lilleholt, 2018; Shen et al., 2019), the current study offers important contributions that build on previous work in two distinct ways.

First, this study extends the literature by being the first to use RWA to determine the relative importance of performance expectancy, effort expectancy, and social influence to understanding technology acceptance. Other approaches examining shared variance in a model do not sufficiently test the relative importance of the included variables because they do not take shared variance into account. As suggested by the UTAUT, all three predictors matter, and were significantly correlated with both indices of VR acceptance in this study. Additionally, all three antecedents significantly predicted behavioral intention to use VR; however, only performance expectancy uniquely contributed to the prediction of preference for VR over a video-based alternative to learn a new task. Performance expectancy appears to matter the most when asking students about VR for learning. While not completely supported by all metrics, this question was tested in several ways, and the preponderance of evidence pointed to performance expectancy as the most influential predictor of overall technology acceptance. This supports Davis et al's (1989) previous assertion that performance expectancy is more important that effort expectancy.

These findings might also then support the reasoning that perceived usefulness and ease of use are conceptually similar to instrumentality and expectancy in Vroom's (1964) expectancy theory of motivation. Expectancy theory postulates that motivation is, inpart, a function of instrumentality and expectancy, but instrumentality is concerned with the relationship between performing a behavior and obtaining a desired outcome. Thus, like performance expectancy, instrumentality is more proximal to expectations of a desired outcome. Students who perceive that using VR will increase their learning are especially likely to embrace this medium. In cost-benefit terms, it seems that students are most willing to expend limited resources (e.g., effort) on VR technology when it will pay off with respect to performance gains.

Venkatesh et al. (2003) do not suggest that effort expectancy or social influence are stronger than the other, and that is reflected in our results. They suggest that certain important moderators, such as the voluntariness of usage and prior experience with the technology, might influence these findings. Given that our analyses did not find a relationship between VR experience and behavioral intention to use VR or preference for $\mathrm{VR}$, more research will be needed on the role of these variables as moderators when examining acceptance of VR for learning.

Second, the study also uniquely measures technology acceptance in two distinct ways. Most technology acceptance studies measure intentions through Likert-type ratings. While such ratings provide useful information, this practice is not without limitations. Such an assessment shares common method variance with Likert-type ratings of antecedents such as performance expectancy, effort expectancy, and social influence (see Podsakoff et al., 2003). Including an additional measure of technology acceptance not measured via Likert-type ratings offers an opportunity to assess the robustness of theories like UTAUT across different measurement techniques. The present study suggests 
that asking people to report their preference for VR over an alternative is a viable way to quickly gauge technology acceptance. This offers researchers and practitioners a relatively easy way to measure technology acceptance, which provides a related but different view of how people feel about using an emerging technology such as VR.

Another important consideration is why students might have preferred VR instead of YouTube. In many ways, the answer might be found in the greater weight placed upon the importance of performance expectancy. The question that students appear to be asking themselves when choosing between audiovisual forms of learning is: "which one will better teach me what I need to know?" This explanation is plausible given that procedural knowledge is typically gained through practice (Kraiger et al., 1993), which is easier facilitated through interactive VR than observable video. Perhaps students feel a technology which enables them to practice as they learn is superior to one that does not. For some, the novelty of VR may have also contributed to its appeal. Perhaps some students gravitated toward VR because it is newer and believed to be more innovative. Future research could explore whether the perceived novelty of a learning tool such as VR contributes to technology acceptance, and if so whether that effect is moderated by situational variables such as the "stakes" or consequences of learning errors and individual differences such as openness to experience and goal orientation.

\section{Practical implications}

There is reason to believe that post-secondary students may not be taking full advantage of VR resources available to them. The current study itself provides evidence that even with a broad range of VR options on campus, approximately half of the participants did not identify prior VR experience. Thus, value exists in understanding what matters, as well as what matters most, to post-secondary students considering whether to leverage VR resources on campus. Discovering the characteristics that influence whether students choose VR to learn can assist post-secondary institutions in making decisions regarding investment in VR technology, as well as provide insight into what data would be important to collect from student populations prior to implementation. This will allow post-secondary institutions to examine a priori students' acceptance of VR before devoting time and resources into the development of VR programs or software. Knowing what factors influence whether an individual gravitates toward and eventually accepts VR for learning can inform the development of interventions that help students gain comfort with VR and gathering this information does not require that students have had prior experience with VR. Understanding what beliefs predict acceptance of VR for learning allows post-secondary institutions to decide whether, under what conditions, how, and for whom to deploy VR for learning.

In a sense, each of the three predictor variables included in this study can be viewed as "levers," which may work to increase or decrease technology acceptance depending on their individual and collective setpoints. If post-secondary institutions find themselves in a position where students' performance expectancy, effort expectancy, and social influence are all low, it is crucial to understand which of these levers will have the greatest effect. The current study provides evidence that post-secondary institutions seeking to prepare students for VR may wish to begin by emphasizing the positive outcomes associated with VR for learning and its effectiveness in helping students grasp new 
concepts. Students who see VR as performance enhancing are especially likely to want to use it. This notion is consistent with other research streams pointing to the importance of enhancing expectations of outcomes to achieve behavioral change (Bandura, 1997; Schunk, 2012; Wigfield et al., 2009). For example, Thomas and Velthouse's (1990) cognitive model of empowerment suggests that successful empowerment interventions should aim to improve task assessments, which are rooted in part in perceptions of performance expectancy-type constructs. Furthermore, research has shown that expectancies are strong predictors of student choices and are related with cognitive engagement and achievement (e.g., Schunk et al., 2008).

When possible, post-secondary institutions should consider performance expectancy, effort expectancy, and social influence simultaneously, in addition to other important individual differences such as motivation, enjoyment, and presence (Makransky \& Lilleholt, 2018). By collecting student data on a variety of antecedents to preference for VR, campus decision makers can make more informed decisions about the direction and target of interventions aimed at easing the implementation of VR for learning. For example, testimonials evoking a sense of social influence could be useful in getting students to give VR labs a try.

\section{Limitations and future directions}

This study's findings should not be used to justify the utilization of VR as the sole method of learning, nor to suggest that the UTAUT predictors are the only variables that explain VR acceptance on campus. Instead, these findings provide a starting point for understanding what shapes how students feel about using VR to learn. Additional research is needed to expand upon this study's findings and examine whether the results replicate under different circumstances. For example, the current study involved learning a procedural task; future research should examine whether this study's results generalize to learning opportunities that involve the acquisition of declarative knowledge rather than procedural skills.

The nature of our research design limited us to selecting only one task for students to consider when deciding on VR or video as a training medium. Given that suturing is a task which involves piercing skin and potentially bodily fluids, it is possible that some students preferred video over VR simply to avoid more immersive exposure to images that could be considered off-putting. However, we did not explicitly state that either the video or VR training would show bodily fluids or human anatomy. Students were left to form their own interpretation of how the training material would be presented. They could imagine the training video depicting the suturing of a real person or a training dummy. Similarly, they could imagine the VR training depicting a hyper-realistic, virtual person or on an unrealistic, virtual dummy. Thus, we do not expect that the sensitive, medical nature of the suturing task inherently drove students to one training medium over the other, as suggested by the substantial variance in the preference variable. However, this was not directly tested. Future studies should examine whether the nature of the task in question changes students' acceptance of VR for learning.

Future studies should also expand on the current methodology, for example, by examining acceptance of VR for learning when there are greater stakes than the norisk learning task presented in this research. It is also important to point out that 
the current study measured acceptance of VR through intentions and preferences; however, actual behaviors were not assessed. While behavioral outcomes are indeed beneficial to measure in their own right, the measurement of acceptance of VR is not contingent on a population having made use of VR previously and thus was not critical to the execution of this study. Future research should take this line of investigation a step further by actually giving students the option of using VR or video for a learning task and then measuring their behavior (e.g., Who shows up at the VR lab? How long do they stay?) as well as their learning outcomes and reactions to the experience.

Additional boundary conditions should also be considered. This study was designed to speak to the discretionary utilization of VR-that is, students' interest in using VR to learn when they do not have to, and when alternatives are available. In the parlance of social psychology, this constitutes a "weak situation" where a prescribed course of action is not particularly well defined. While not the focus of this study, students' usage patterns in "stronger" situations, such as when VR is actively introduced in a class, are also worthy of continued investigation.

Another important limitation is that our study only compared VR to video as a comparable medium of learning. To facilitate this comparison, we described each learning option in the form of an informational article, which is shown in Appendix A. We attempted to describe both options in a manner representative of how they would be described in public discourse while not presenting a clear favorability of one over the other. However, the favorability of wording between the descriptions was not identical. VR was presented as a "newer" option, and it was noted that it has many benefits. We cannot rule out the possibility that our descriptions of the two options as shown in Appendix A article influenced the percentage of people who chose video vs. VR. We have no reason to believe this would have influenced the relationships among the study variables.

We chose video training as an alternative because it is a common, familiar learning method, which is often utilized in situations where the use of VR may be alternatively suggested. However, it is entirely possible that some students prefer more familiar methods of learning (e.g., lecture) over VR and video. Future research should examine the degree to which students opt for VR when given other learning medium alternatives, such as lectures, seminars, games, role plays, and augmented reality. In the present study, some students' preference for VR may have reflected more of a dislike for video (or specifically YouTube) as a form of learning than a high level of enthusiasm for VR. To mitigate this concern, behavioral intention to use VR assessed acceptance of VR independent of an alternative, such that students were asked to report their likelihood of using VR if implemented at their university without comparing it to another training method.

Finally, there are other aspects of the UTAUT. The original UTAUT model includes facilitating conditions as a predictor of behavioral usage (Venkatesh et al., 2003), and other studies have integrated a range of additional factors and moderators into the model (see Venkatesh et al., 2016). Future research should explore other aspects of the UTAUT, as well as whether the relative weights found in this study hold up within other technology acceptance contexts. 


\section{Conclusion}

This study presents support for key psychological variables that predict students' acceptance of VR for learning, and thus provides a framework that can help postsecondary institutions make more informed decisions about VR technologies for their campuses. By surveying students' performance expectancy, effort expectancy, and social influence- and by extension evaluating students' beliefs on utilizing VR for learning- post-secondary institutions can get a better perspective on how implementing new VR technologies would be received on their campuses and where expectations could be strengthened.

\section{Appendix A}

\section{Training methods}

Lifelong learning is more important now than ever before. These days, there are a wide variety of training methods available to organizations training employees, as well as individuals seeking to learn new things. For example, lectures, on-the-job training, near-the-job training, and apprenticeships are all options for individuals to learn new skills. This article focuses on two particular options which have become increasingly popular with the advancement of technology: (a) instructional videos posted on YouTube, and (b) virtual reality training.

\section{YouTube}

YouTube as a learning tool has become increasingly common in training research. There are many instructional videos available on YouTube today, and YouTube can be a useful training tool for a variety of different topics. For example, people can learn mechanical and motor skills (for example, welding, stitching, or cooking) or interpersonal skills (for example, interviewing, customer service, or public speaking) by watching YouTube videos posted online. Typically, these videos will provide an instructor who talks the learner through core concepts, and also demonstrates (or models) how to accomplish the training task. For instance, a YouTube video teaching trainees how to give an insulin shot to a diabetic patient may show the instructor presenting all the necessary materials (i.e., alcohol prep pap, insulin, needle), then demonstrating step-by-step how to prepare the injection site and administer the shot on a person. These videos allow learners to pause and repeat any information that is not clear or watch several times to ensure the knowledge is retained.

\section{VR}

A newer method of workplace training is Virtual Reality (VR). Virtual reality is another popular training method. Modern VR systems include a headset and two hand-held devices which immerse an individual in a virtual simulation and allow the individual to navigate and interact with objects in that virtual simulation. VR training is conducted by putting trainees into the headset and allowing them to experience a task firsthand in a simulated environment. People can use VR training to learn interpersonal skills (for example, interviewing, customer service, or public speaking) or 
mechanical and motor skills (for example, welding, stitching, or cooking). For example, a VR simulation teaching individuals to use an insulin shot might place them in a scenario where they could access all the necessary materials (i.e., alcohol prep pad, insulin, needle), and a narrator would speak aloud to guide the trainee step-by-step as the trainee uses the materials to administer the shot to a virtual person. The benefits to VR training are many. In the insulin shot example, individuals are able to practice inserting the needle and would receive immediate feedback as to whether the insertion was too much, too little, or just right. Furthermore, individuals are able to prompt the narrator to repeat instructions simply by aiming and clicking a button on the controller.

\section{Conclusion}

Both training methods share similarities with respect to the information presented, but they present the information in different ways. No matter the training method, the learner's knowledge or skill acquisition is typically tested after the training is complete, to ensure mastery. For example, those being taught to administer insulin shots may be given a test after completing the YouTube or VR training. This test would likely give trainees the opportunity to practice giving shots on a life-like dummy. Such a test allows employers and learners alike to see if the learners have acquired the new knowledge and/or mastered the new skill.

\section{Appendix B}

Preference for VR

Imagine coming to campus to learn how to complete a new task. The task you'll be asked to learn is one that surgeons do, called suturing. Suturing, which is when surgeons stitch up an incision on the body, is a basic skill all surgeons need to learn. The task requires technical knowledge which can be learned through either VR or YouTube learning methods. Imagine you will be given the opportunity to learn in our laboratory using one of two methods (VR or YouTube) for however long you would like. We can teach you how to use the VR system or how to properly search YouTube for videos if you would like. Once you are comfortable with your method of choice, you have the opportunity to use that method to learn the suturing task for as long as necessary. When you finish practicing, we will give you a training dummy to perform the suture on. The amount of time it takes to complete this task on the dummy will be measured and compared to other participants. Accuracy will also be measured, and final performance will be scored according to both time and accuracy.

Please choose which method you would like to use to learn how to complete the task:

Interactive VR program which provides instruction and practice of the task.

Access to YouTube video examples demonstrating detailed completion of the task. 


\section{Appendix C}

\section{UTAUT scale (adapted)}

Participants select one response to each item utilizing a seven-point Likert scale, from Strongly Disagree (1) to Strongly Agree (7).

Imagine [university] starts a training program for students. The program gives students access to VR technologies to train new skills. Please respond to each of the following items with that scenario in mind.

\section{Performance expectancy}

1. I would find virtual reality (VR) useful in training.

2. Using VR enables me to accomplish tasks more quickly.

3. Using VR increases my productivity.

4. If I use VR, I will increase my chances of learning.

\section{Effort expectancy}

1. My interaction with VR would be clear and understandable.

2. It would be easy for me to become skillful at using VR.

3. I would find VR easy to use.

4. Learning to operate VR would be easy for me.

\section{Social influence}

1. People who influence my behavior think that I should use VR for learning.

2. People who are important to me think that I should use VR for learning.

3. The [university] administration has been helpful in the use of VR for learning.

4. In general, [university] has supported the use of VR for learning.

\section{Behavioral Intention to use VR for learning}

1. I would intend to use the VR system for learning in the first three months.

2. I predict I would use the VR system for learning in the first three months.

3. I would plan to use the VR system for learning in the first three months. 


\section{Acknowledgements}

We would like to thank Chelsi Campbell and Adam W. Meade for their assistance on this project.

\section{Authors' contributions}

SMN: conceptualization, methodology, formal analysis, investigation, resources, data curation, Writing —original draft, writing - review and editing, visualization, project administration, JDS: Conceptualization, methodology, validation, resources, writing—original draft, writing—review and editing, LLF: conceptualization, methodology, writing—original draft, writing—review and editing. All authors read and approved the final manuscript.

\section{Funding}

Not applicable.

\section{Availability of data and materials}

The datasets generated and analyzed during the current study are not publicly available due to participant confidentiality but are available from the corresponding author on reasonable request.

\section{Declarations}

\section{Competing interests}

The authors declare that they have no competing interests.

Received: 25 June 2021 Accepted: 7 December 2021

Published online: 31 January 2022

\section{References}

Ajzen, I. (1991). The theory of planned behavior. Organizational Behavior and Human Decision Processes, 50, $179-211$. https://doi.org/10.1016/0749-5978(91)90020-T

Amirian, M. J., Lindner, S. M., Trabulsi, E. J., \& Lallas, C. D. (2014). Surgical suturing training with virtual reality simulation versus dry lab practice: An evaluation of performance improvement, content, and face validity. Journal of Robotic Surgery, 8(4), 329-335. https://doi.org/10.1007/s11701-014-0475-y

Bandura, A. (1997). Self-efficacy: The exercise of control. W. H. Freeman and Company.

Bandura, A. (1977). Social learning theory. Prentice Hall. https://doi.org/10.1177/105960117700200317

HTC Corporation (2021). Vive. Vive. https://www.vive.com/us/.

Cortina, J. M. (1993). What is coefficient alpha? An examination of theory and applications. Journal of Applied Psychology, 78(1), 98-104. https://doi.org/10.1037/0021-9010.78.1.98

Davis, F. D. (1989). Perceived usefulness, perceived ease of use, and user acceptance of information technology. MIS Quarterly, 13, 319-339. https://doi.org/10.2307/249008

Davis, F. D., Bagozzi, R. P., \& Warshaw, P. R. (1989). User acceptance of computer technology: A comparison of two theoretical models. Management Science, 35, 982-1003. https://doi.org/10.1287/mnsc.35.8.982

Dwivedi Y. K., Rana N. P., Chen H., Williams M. D. (2011) A meta-analysis of the Unified Theory of Acceptance and Use of Technology (UTAUT). In M. Nüttgens, A. Gadatsch, K. Kautz, I. Schirmer, N. Blinn (Eds.) Governance and sustainability in information systems. Managing the transfer and diffusion of IT. (pp. 155-170). Springer. https://doi.org/10.1007/978-3642-24148-2_10.

FSR Education. (2018, March 16). Use case: VR technology at a community college. FSR. https://fsrinc.com/education/colla borate-ed-tech-blog/117-use-case-vr-technology-at-a-community-college.

Forest, C. R., Moore, R. A., Jariwala, A. S., Fasse, B. B., Linsey, J., Newstetter, W., Ngo, P., \& Quintero, C. (2014). The invention studio: A university maker space and culture. Advances in Engineering Education, 4, 1-32.

Gupta, B., Dasgupta, S., \& Gupta, A. (2008). Adoption of ICT in a government organization in a developing country: An empirical study. The Journal of Strategic Information Systems, 17, 140-154. https://doi.org/10.1016/j.jsis.2007.12.004

Hobfoll, S. E. (1989). Conservation of resources: A new attempt at conceptualizing stress. American Psychologist, $44,513$. https://doi.org/10.1037/0003-066X.44.3.513

Howard, M. C. (2018). Virtual reality interventions for personal development: A meta-analysis of hardware and software. Human-computer Interaction, 34, 205-239. https://doi.org/10.1080/07370024.2018.1469408

Jensen, L., \& Konradsen, F. (2018). A review of the use of virtual reality head-mounted displays in education and training. Education and Information Technologies, 23, 1515-1529. https://doi.org/10.1007/s10639-017-9676-0

Johnson, J. W. (2000). A heuristic method for estimating the relative weight of predictor variables in multiple regression. Multivariate Behavioral Research, 35, 1-19. https://doi.org/10.1207/S15327906MBR3501_1

Jones, N. (2018). Simulated labs are booming. Nature, 562(7725), S5-S7. https://doi.org/10.1038/d41586-018-06831-1

Kazemi, H., Rappel, J. K., Poston, T., Hai Lim, B., Burdet, E., \& Leong Teo, C. (2010). Assessing suturing techniques using a virtual reality surgical simulator. Microsurgery, 30,479-486. https://doi.org/10.1002/micr.20766

Kraiger, K., Ford, J. K., \& Salas, E. (1993). Application of cognitive, skill-based, and affective theories of learning outcomes to new methods of training evaluation. Journal of Applied Psychology, 78, 311-328. https://doi.org/10.1037/0021-9010. 78.2 .311

Le, B. (2018, March 9). Virtual reality and augmented reality among new majors offered to Chapman students. Chapman University. https://news.chapman.edu/2018/03/09/virtual-reality-and-augmented-reality-among-new-majors-offer ed-to-chapman-students/. 
Makransky, G., Borre-Gude, S., \& Mayer, R. E. (2019). Motivational and cognitive benefits of training in immersive virtual reality based on multiple assessments. Journal of Computer Assisted Learning, 35, 691-707. https://doi.org/10.1111/ jcal. 12375

Makransky, G., \& Lilleholt, L. (2018). A structural equation modeling investigation of the emotional value of immersive virtual reality in education. Educational Technology Research and Development, 66, 1141-1164. https://doi.org/10 1007/s11423-018-9581-2

Man, D. W. (2018). Virtual reality-based cognitive training for drug abusers: A randomised controlled trial. Neuropsychological Rehabilitation, 30(2), 315-332. https://doi.org/10.1080/09602011.2018.1468271.

Margaryan, A., Littlejohn, A., \& Vojt, G. (2011). Are digital natives a myth or reality? University students' use of digital technologies. Computers and Education, 56, 429-440. https://doi.org/10.1016/j.compedu.2010.09.004

Meade, A. W., \& Craig, S. B. (2012). Identifying careless responses in survey data. Psychological Methods, 17, 437. https://doi. org/10.1037/a0028085

Oculus VR, LLC (2021). Oculus. Oculus. https://www.oculus.com/.

Pak, R., Rovira, E., McLaughlin, A. C., \& Leidheiser, W. (2017). Evaluating attitudes and experience with emerging technology in cadets and civilian undergraduates. Military Psychology, 29(5), 448-455. https://doi.org/10.1037/mil0000175

Podsakoff, P. M., MacKenzie, S. B., Lee, J. Y., \& Podsakoff, N. P. (2003). Common method biases in behavioral research: A critical review of the literature and recommended remedies. Journal of Applied Psychology, 88(5), 879. https://doi.org/10. 1037/0021-9010.88.5.879.

Radianti, J., Majchrzak, T. A., Fromm, J., \& Wohlgenannt, I. (2020). A systematic review of immersive virtual reality applications for higher education: Design elements, lessons learned, and research agenda. Computers and Education, 147, 103778. https://doi.org/10.1016/j.compedu.2019.103778

Rupp, M. A., Odette, K. L., Kozachuk, J., Michaelis, J. R., Smither, J. A., \& McConnel, D. S. (2019). Investigating learning outcomes and subjective experiences in 360-degree videos. Computers and Education, 128, 256-268. https://doi.org/10. 1016/j.compedu.2018.09.015

Samadbeik, M., Yaaghobi, D., Bastani, P., Abhari, S., Rezaee, R., \& Garavand, A. (2018). The applications of virtual reality technology in medical groups teaching. Journal of Advances in Medical Education and Professionalism, 6, 123-129. PMID: 30013996.

Schaffhauser, D. (2019, May 15). 9 amazing uses for VR and AR in college classrooms. Campus Technology. https://campu stechnology.com/Articles/2019/05/15/9-Amazing-Uses-for-VR-and-AR-in-College-Classrooms.aspx?Page=1.

Schunk, D. H., Pintrich, P. R., \& Meece, J. L. (2008). Motivation in education: Theory, research, and applications (3rd ed.). Pearson Education.

Schunk, D. H. (2012). Learning theories an educational perspective sixth edition. Pearson.

Shen, C. W., Ho, J. T., Ly, P.T. M., \& Kuo, T. C. (2019). Behavioural intentions of using virtual reality in learning: Perspectives of acceptance of information technology and learning style. Virtual Reality, 23, 313-324. https://doi.org/10.1007/ s10055-018-0348-1

Siu, K. C., Best, B. J., Kim, J. K., Oleynikov, D., \& Ritter, F. E. (2016). Adaptive virtual reality training to optimize military medical skills acquisition and retention. Military Medicine, 181, 214-220. https://doi.org/10.7205/milmed-d-15-00164

Thomas, K. W., \& Velthouse, B. A. (1990). Cognitive elements of empowerment: An "interpretive" model of intrinsic task motivation. Academy of Management Review, 15, 666-681. https://doi.org/10.2307/258687

Tonidandel, S., \& LeBreton, J. M. (2015). RWA web: A free, comprehensive, web-based, and user-friendly tool for relative weight analyses. Journal of Business and Psychology, 30, 207-216. https://doi.org/10.1007/s10869-014-9351-z

Tonidandel, S., LeBreton, J. M., \& Johnson, J. W. (2009). Determining the statistical significance of relative weights. Psychological Methods, 14(4), 387. https://doi.org/10.1037/a0017735

University of Washington (2021). UW Reality Lab. UW Reality Lab. https://realitylab.uw.edu/.

Venkatesh, V., \& Davis, F. D. (2000). A theoretical extension of the technology acceptance model: Four longitudinal field studies. Management Science, 46, 186-204. https://doi.org/10.1287/mnsc.46.2.186.11926

Venkatesh, V., Morris, M. G., Davis, G. B., \& Davis, F. D. (2003). User acceptance of information technology: Toward a unified view. MIS Quarterly, 27, 425-478. https://doi.org/10.2307/30036540

Venkatesh, V., Thong, J. Y. L., \& Xu, X. (2016). Unified theory of acceptance and use of technology: A synthesis and the road ahead. Journal of the Association for Information Systems, 17, 328-376. https://doi.org/10.17705/1jais.00428

Vroom, V. H. (1964). Work and motivation. Wiley.

Weirs, A. (2020, May) Three ways people are using YouTube to learn at home during the coronavirus pandemic. ThinkWithGoogle. https://www.thinkwithgoogle.com/consumer-insights/consumer-trends/how-people-use-youtube-forlearning/.

Wigfield, A. Tonks, S., \& Klauda, S. L. (2009). Expectancy value theory. In K. R. Wentzel \& A. Wigfield (Eds.), Handbook of motivation at school (pp. 55-76). Routledge.

Zielinski, D. (2021, March 8) The growing impact of virtual reality on training. SHRM. https://www.shrm.org/hr-today/news/ hr-magazine/spring2021/pages/virtual-reality-training-spreads-its-wings.aspx.

\section{Publisher's Note}

Springer Nature remains neutral with regard to jurisdictional claims in published maps and institutional affiliations. 